\title{
Quantitative Ultrasound Assessment of HIFU Induced Lesions in Rodent Liver
}

\author{
Jeremy Kemmerer, Goutam Ghoshal, and Michael Oelze \\ Bioacoustics Research Laboratory \\ Department of Electrical and Computer Engineering \\ University of Illinois at Urbana-Champaign \\ Urbana, IL 61801 \\ Email:kemmere1@,illinois.edu
}

\begin{abstract}
Quantitative ultrasound (QUS) techniques have been used for tissue characterization by discriminating between tissues of differing microstructure. High Intensity Focused Ultrasound (HIFU) is a means for minimal or non-invasive therapy, which results in changes in tissue microstructure. However, significant challenges remain for the monitoring and assessment of HIFU therapy. Conventional B-mode imaging is not reliable for detection and assessment of thermal lesions produced by HIFU. Because of QUS sensitivity to microstructure changes, it was hypothesized that QUS could assess thermal lesions. A $1-\mathrm{MHz}$ single-element focused transducer (f/1.1) was used to produce thermal lesions in ex-vivo rat liver. The focal intensities used to generate lesions were measured to be between 1500 and 2500 $\mathrm{W} / \mathrm{cm}^{2}$ in degassed water using a calibrated needle hydrophone. These exposures were monitored using a clinical ultrasound array (L14-5, Ultrasonix). Treated and untreated regions as determined by histology and visual inspection were compared to parametric images of QUS parameters (Effective Scatterer Diameter (ESD), and Effective Acoustic Concentration (EAC)). An average increase in ESD of $15 \%$ was observed in three samples, while EAC estimates decreased by $4.3 \mathrm{~dB}$. These findings suggest that QUS can be used for non-invasive assessment of thermal lesions.
\end{abstract} HIFU;

Keywords: Quantiative ultrasound; Tissue characterization;

\section{INTRODUCTION}

Ultrasound has been used clinically for decades, and yet the full potential of ultrasound to non-invasively image and characterize tissues is still being realized. Quantitative ultrasound (QUS), which utilizes the RF signal spectrum from backscattered ultrasound, continues to present a promising extension of more traditional B-mode imaging in medical applications. Early work in opthamology [1] showed the potential value of analyzing the RF spectrum for diagnostic purposes. Since then, QUS techniques have been successfully demonstrated for use in the prostate [2], breast cancer [3-5], and detection of metastases in lymph nodes [6].

QUS research efforts also extend beyond pulse-echo measurements and into applications for therapy. QUS approaches are being investigated for monitoring of thermal therapy [7], and high frequency ultrasound has been demonstrated to be sensitive to detection of cellular apoptosis [8] induced by chemotherapy.

High Intensity Focused Ultrasound (HIFU) has been investigated as a means to non-invasively deliver thermal and mechanical energy to tissues for therapeutic purposes. Early work in HIFU probed the brain and central nervous system [9]. HIFU has since demonstrated promise for the treatment of solid tumors. Specifically, growth of mouse tumors has been interrupted by HIFU exposure [10]. Challenges remain in monitoring and assessment of HIFU therapy. Ultrasound is safe, inexpensive, and provides high spatial and temporal resolution, and so QUS could provide an attractive means for monitoring HIFU. In this work, QUS techniques are applied in rat liver samples to assess lesions induced by HIFU therapy.

\section{METHODS}

\section{A. HIFU Exposure of Liver Samples}

Agar phantoms containing a single lobe of rat liver were exposed to a single-element $1-\mathrm{MHz}(\mathrm{f} / 1.1)$ transducer powered by an A150 $55 \mathrm{~dB}$ power amplifier (ENI, Rochester, NY) and excited by an arbitrary waveform generator (HP 33120a, Agilent Technologies, Santa Clara, CA). The exposure was monitored using a Sonix RP clinical ultrasound system (Ultrasonix, Richmond, BC, Canada) with an L14-5/38 probe sampled at $40 \mathrm{MHz}$. The probe had a nominal center frequency of $7.5 \mathrm{MHz}$. The array probe and single element HIFU transducer were aligned by creating a single lesion in an agar and egg-white phantom with the HIFU system, and aligning the array transducer to this lesion in the B-mode display. The array probe and HIFU transducer were thus aligned and remained fixed throughout the experiment. The location of this lesion was marked on the Sonix RP display and used for targeting in the HIFU liver experiment. The experimental setup, including transducer and sample orientation, is shown in Figure 1.

Sponsored by NIH R01-EB008992 


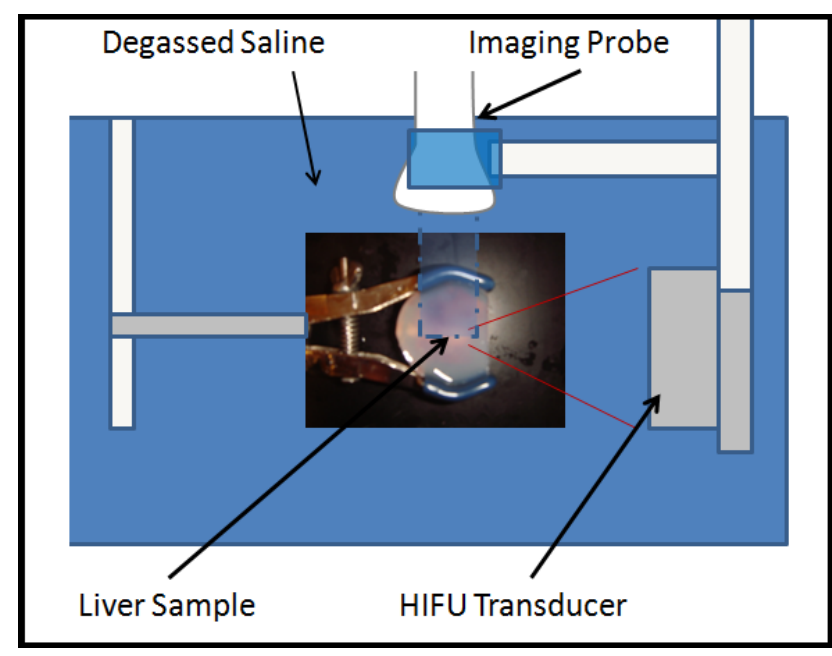

Figure 1 - HIFU Exposure Experimental Setup

Excitation amplitude and duration of the exposure were chosen based on trial and error such that lesions were formed which were visible upon sample inspection. Exposure parameters varied from 10-20 seconds and intensities between 1500 and $2500 \mathrm{~W} / \mathrm{cm}^{2}$, as measured in degassed water using a needle hydrophone (Precision Acoustics, Dorchester, UK). Data were collected during the exposure by synchronizing the capture of the RF data with periods between exposure tonebursts. Increased echogenicity at the focus after exposure in the liver was observed in B-mode images from the Sonix RP in the cases of higher intensity and longer exposure, which was hypothesized to result from boiling and/or cavitation activity. For exposures where this increase in brightness in the B-mode images was not observed, a primarily thermal mechanism for lesion production was assumed.

\section{B. Ultrasonic Assessment of Liver}

Three rat liver samples were scanned with a $20-\mathrm{MHz}$ single-element transducer excited using a Panametrics 5900 pulser-receiver (Olympus NDT, Waltham, MA) and connected to a PC A/D card with $250 \mathrm{MHz}$ sampling. Slices were imaged with spacing of scan lines of approximately $1 / 2$ beamwidth (125 $\mu \mathrm{m}$ for $20 \mathrm{MHz} \mathrm{f} / 3$ ), from which parametric QUS images were generated. Transducer position was controlled using a Daedal positioning system (Daedal, Inc., Harrisburg, PA) through a PC running custom LabView (National Instruments, Austin, TX) software.

In order to determine changes in QUS estimates in liver tissue with exposure to HIFU, single element scans were taken in each liver sample centered at the approximate location of the HIFU transducer focus both before and after HIFU exposure. Photographs were taken of sliced sections of the exposed samples after fixing in $10 \%$ formalin for 2-3 days. The photographs, which show a clear change in color in the area of exposure, were manually registered with the QUS images in order to determine the area of treatment.

Reference scans were collected for the measurement system for QUS analysis to remove instrument-dependency from the resulting estimates of the backscatter coefficient (BSC).
Estimates for BSC were generated for each of several regions of interest (ROIs) contained within a sample. Estimates of the effective scatterer diameter (ESD) and effective acoustic concentration (EAC) were obtained by assuming a spherical Gaussian form factor model [11]. Parametric images were generated for each image slice by overlaying pixels corresponding to ROIs spatially with colors corresponding to computed QUS estimates.

The normalized power spectrum for this approach is given by [11]:

$$
W(f)=\frac{185 L q^{2} a_{e f f}^{6} \rho z_{v a r}^{2} f^{4}}{\left[1+2.66\left(f q a_{e f f}\right]\right.} \exp \left[-12.159 f^{2} a_{e f f}^{2}\right]
$$

where $L$ is the gate length $(\mathrm{mm}), q$ is the ratio of aperture radius to distance from the ROI, $f$ is the frequency $(\mathrm{MHz})$, and $a_{\text {eff }}$ is the effective scatterer radius. The effective acoustic concentration (EAC) term, $\rho z_{\text {var }}{ }^{2}$, represents the product of scattering strength and the number density of scatterers. The scattering strength is the square of the impedance mismatch between scatterer and background divided by the background impedance.

\section{RESULTS}

Fresh rat liver samples were scanned using the high frequency ultrasound system before and after exposure to HIFU around the spatial location of the HIFU transducer focus, yielding correlated before and after parametric QUS images. Example ESD, EAC, and B-mode images of a sample of rat liver after exposure are shown in Figure 2, along with a photograph of the same sample. The ESD increases in the lesion region, while the location of changes in EAC generally appears below the region designated as lesion.
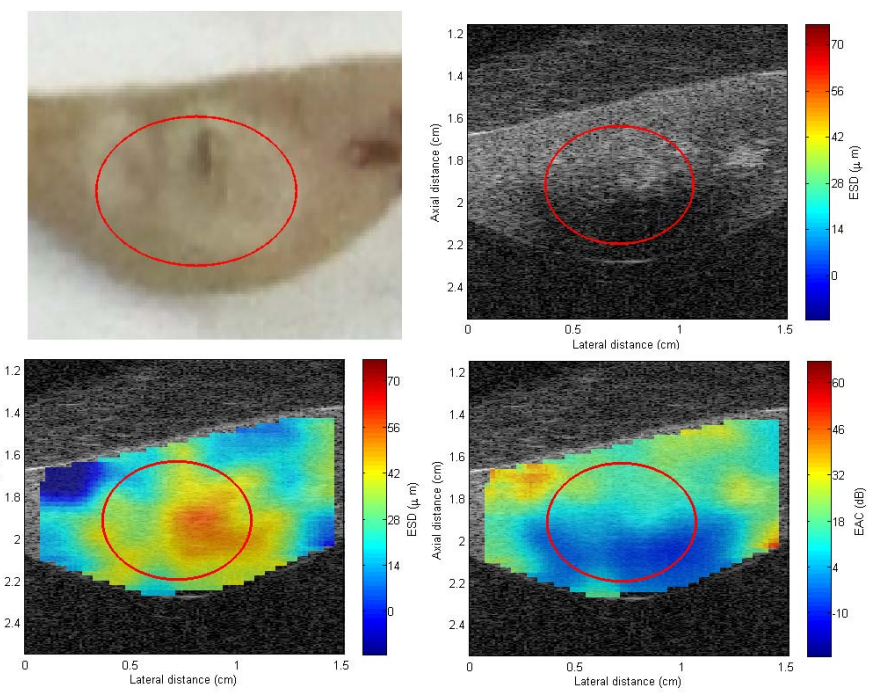

Figure 2 - (Clockwise from top left): Photograph of treated rat liver, B-mode, EAC parametric image, and ESD parametric image.

Quantitative comparison of estimates in regions designated as "lesion" before and after exposure further demonstrates 
changes in both ESD and EAC with treatment. Figure 3 shows an example map of scatterer property estimates directly on the location of the lesion before exposure. ESD estimates were observed to increase by an average of $15 \%$ after treatment, while EAC was found to decrease by $4.3 \mathrm{~dB}$.

A total of three fresh rat liver samples were examined in this study. A second set of images from a rat liver are shown in Figure 4. Similar to the first set of data in figure 2, the second set of images also show the ESD increasing due to the thermal lesion and the EAC decreasing due to the thermal lesion. A summary of the experimental results from three rat livers is shown in Table 1. Statistically significant differences were observed using analysis of the variance (ANOVA, $\mathrm{p}<0.05$ ) between ESD and EAC estimates for each liver sample tested. However, it should be noted from Table I that the variances for the ESD and EAC estimates were relatively large. A feature analysis plot (Figure 5) shows clear separation of average QUS parameters before and after heating for three samples. Therefore, it is expected that regression analysis could provide characterization of lesions because minimal overlap occurred between heated versus unheated samples.
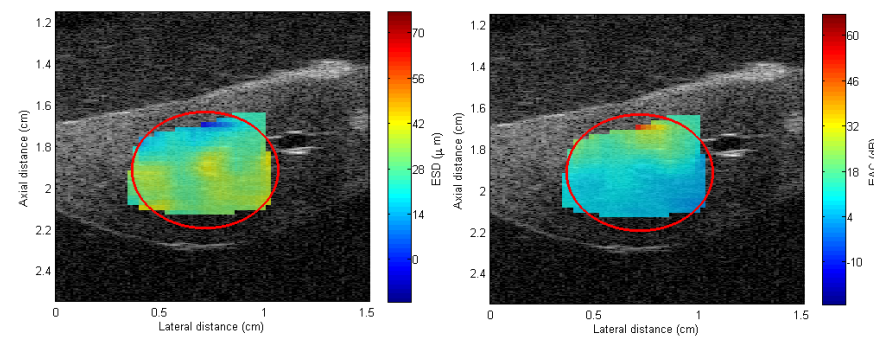

Figure 3 - ROI selection for quantitative comparison (red circle indicates location of visible lesion)
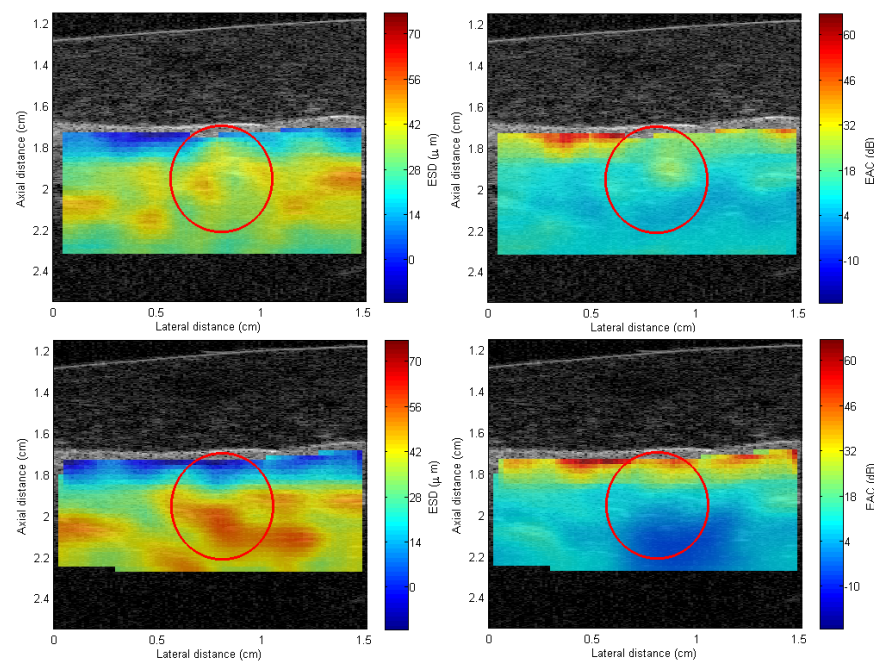

Figure 4 - ESD and EAC of a liver sample before (top) and after (bottom) HIFU exposure

\section{CONCLUSIONS}

Conventional B-mode images have proven to be inadequate for monitoring and accessing HIFU treatment. QUS parameters have been observed to provide sensitivity to changes in tissue structure induced by HIFU. ESD parameters were found to increase by an average of $15 \%$ in treated compared to untreated regions, while EAC was found to decrease by $4.3 \mathrm{~dB}$.

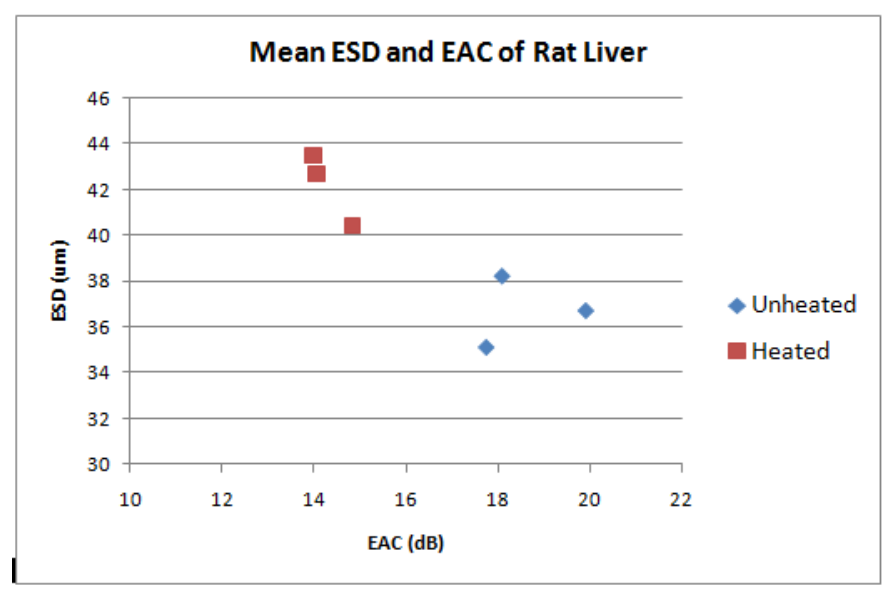

Figure 5 - Feature analysis plot of rat liver QUS estimates

TABLE I. ESD AND EAC FROM LIVER SAMPLES BEFORE AND AFTER HIFU EXPOSURE

\begin{tabular}{|l|c|c|c|c|}
\hline \multirow{2}{*}{ Animal \# } & \multicolumn{2}{|c|}{ ESD (um) } & \multicolumn{2}{c|}{ EAC (dB) } \\
\cline { 2 - 5 } & Before & After & Before & After \\
\hline 1 & $35.1 \pm 7.8$ & $42.7 \pm 8.3$ & $17.7 \pm 6.4$ & $14.1 \pm 6.5$ \\
\hline 2 & $36.7 \pm 7.9$ & $43.5 \pm 8.9$ & $19.9 \pm 6.0$ & $14.0 \pm 5.9$ \\
\hline 3 & $38.2 \pm 4.4$ & $40.4 \pm 4.6$ & $18.1 \pm 2.3$ & $14.8 \pm 2.4$ \\
\hline
\end{tabular}

Although changes in QUS parameters were observed in HIFU-induced visible lesions in rat liver, the exact mechanism for theses change remains unclear. Specifically, the observed increase in ESD and decrease in EAC of liver tissue with HIFU treatment may be due to some combination of changes in acoustic scattering generated by tissue microstructure changes, macroscopic damage caused by mechanical stresses, or by acoustic cavitation. The pressures used in the HIFU exposure were in excess of levels for which acoustic cavitation has been observed at $1 \mathrm{MHz}$ [12] in canine muscle. Sudden increases in brightness were observed in the real-time B-mode images used to monitor the experiments in some cases. However, changes in QUS parameters occurred even in cases without this increased brightness, suggesting that QUS is sensitive to other changes in tissue. Future studies will examine histological maps of the damaged tissues in attempts to relate the changes in the QUS parameters with possible microstructural changes due to the thermal injury.

ANOVA analysis revealed statistically significant differences in ESD and EAC for all three samples considered $(p<0.05)$. However, the variance for the ESD and EAC estimates was relatively large. A likely explanation for this large variation was the sensitivity of QUS to macroscopic structures in tissues, such as blood vessels, which can appear in B-mode images as specular scatterers. Because QUS parameters were generated assuming diffuse scattering, measurements from sample regions containing specular 
scatterers may not produce meaningful estimates. Removing ROI segments containing specular scatterers is expected to reduce the variance of the QUS estimates in this data set.

Careful studies relating the degree of change in QUS parameters to exposure dosage may provide more clues to the underlying mechanism of changes in scattering in treated tissues, as well as a useful means to investigate the potential of QUS for HIFU dosimetry. The observed QUS changes with treatment validate the hypothesis that changes induced by HIFU are detectable with QUS. A better understanding of the mechanisms responsible for changes in estimates of scattering in tissue, correlation of changes observed in QUS estimates with changes observed in histological evaluation, and better control of HIFU dose and acoustic cavitation remain key challenges in this effort.

\section{ACKNOWLEDGMENT}

This work was supported by NIH Grant R01-EB008992.

\section{REFERENCES}

[1] E. J. Feleppa, F. L. Lizzi, D. J. Coleman, and M. M. Yaremko, "Diagnostic spectrum analysis in ophthalmology: a physical perspective," Ultrasound Med. Biol., vol. 12, no. 8, pp 623-631, 1986.

[2] E. J. Feleppa et. al., "Typing of prostate tissue by ultrasonic spectrum analysis," IEEE Trans. Ultrason. Ferroelectr. Freq. Control, vol. 43, no. 4, 1996.

[3] M. L. Oelze, W. D. O'Brien, J. P. Blue, and J. F. Zachary, "Differentiation and characterization of rat mammary fibroadenomas and 4T1 mouse carcinomas using quantitative ultrasound imaging," IEEE Trans. Med. Imag., vol. 23 , no. $6,2004$.

[4] M. L. Oelze and J. F. Zachary, "Examination of cancer in mouse models using high-frequency quantitative ultrasound," Ultrasound Med. Biol., vol. 32, no. 11, pp. 1639-1648, 2006.

[5] R. J. Lavarello and M. L. Oelze, "Implementation of scatterer size imaging on an ultrasonic breast tomography scanner," in IEEE International Ultrasonics Symposium Proceedings., Rome, 2009, pp. 305-308.

[6] J. Mamou et. al., "Three-Dimensional High-Frequency Characterization of Cancerous Lymph Nodes," Ultrasound Med. Biol., vol. 36, no. 3, pp 361-375, 2010.

[7] G. Ghoshal and M. Oelze, "Temperature dependent ultrasonic characterization of biological media," submitted for publication.

[8] G. J. Czarnota, M. C. Kolios, J. Abraham, M. Portnoy, F. P. Ottensmeyer, J. W. Hunt, and M. D. Sherar, "Ultrasound imaging of apoptosis: high resolution non-invasive monitoring of programmed cell death in vitro, in situ and in vivo," Br. J. Cancer, vol. 81, no. 3, pp. 520-7, 1999.

[9] J. W. Barnard, W. J. Fry, F. J. Fry, and R. F. Krumins, "Effects of high intensity ultrasound on the central nervous system of the cat," J. Comp. Neurol., vol. 103, pp. 459-484, 1955.

[10] G. ter Haar, I. Rivens, L. Chen, and S. Riddler, "High intensity focused ultrasound for the treatment of rat tumors," Phys. Med. Biol., vol. 36, no. 11, $1495-1501,1991$.
[11] M. L. Oelze, J. F. Zachary, and W. D. O’Brien, “Characterization of tissue microstructure using ultrasonic backscatter: theory and technique for optimization using a Gaussian form factor," J. Acoust. Soc. Amer., vol. 112, pp. 1202-1211, 2002.

[12] K. Hynynen, "The threshold for thermally significant cavitation in dog's thigh muscle in vivo," Ultrasound Med. Biol., vol. 17, no. 2, pp 157-169, 1991. 